\title{
STATIC OUTPUT FEEDBACK CONTROL OF STOCHASTIC DECENTRALIZED SINGULARLY PERTURBED SYSTEMS
}

\author{
Walid Alfuhaid ${ }^{1}$, Saud Alghamdi ${ }^{2}$, John Watkins ${ }^{3}$, M. Edwin Sawan ${ }^{4}$ \\ ${ }^{1,2}$ EECS, Wichita State University, KS, USA \\ ${ }^{3,4}$ Professor, EECS, Wichita State University, KS, USA
}

\begin{abstract}
Designing a controller for stochastic decentralized interconnected large scale systems usually involves a high degree of complexity and computation ability. Noise, observability, and controllability of all system states, connectivity, and channel bandwidth are other constraints to design procedures for distributed large scale systems. The quasi-steady state model investigated in this paper is a reduced order model of the original system using singular perturbation techniques. This paper results in an optimal control synthesis to design an output feedback controller by standard stochastic control theory techniques using Linear Quadratic Regulator (LQR) approach and minimizing a Hamiltonian function with less complexity and computation requirements. Numerical example is given at the end to demonstrate the efficiency of the proposed method.
\end{abstract}

Keywords: Decentralized Control, Singular Perturbation, Static Output, and Optimal Control

\section{INTRODUCTION}

Some systems' setups involve large-scale and highdimension interconnected in such a way as to require huge channel bandwidth for controller communication, complex controller design, and expensive implementation. To overcome such limitation of interconnected large-scale systems, decentralized control setup can simplify controller design and reduce the order of the system, communication channel bandwidth, and implementation cost. The order of interconnected large-scale systems can be reduced using singular perturbation techniques by separating the slow and fast dynamics of the overall system behavior. This reduction can minimize the complexity of control algorithms and implementation cost [1]. Considerable research effort has been concentrated toward singular perturbation for long time and interested many researchers as a mathematical technique to simplify and reduce the order of large-scale control systems [2]-[11].

Interconnected subsystems are controlled separately and independently using decentralized control strategies. Each subsystem can be controlled locally using its own states for state feedback control. This makes controllers communicate locally within each subsystem individually. This technique assumes the availability of all states of each subsystem to the local controller, which is not always applicable in real world systems. Usually, state feedback control techniques are used to control and stabilize an unstable system. In the case when system states are not available, control can be implemented using static output feedback by utilizing the information provided by the output signal to generate the control feedback gain. The unavailability of the states is not the only problem involved in using output feedback techniques; the noise in output measurement and disturbance to controller input can have a considerable effect on system performance and even lead to instability. Stochastic control techniques may be used to describe the system with uncertainties [12]-[14].

Decentralized control is widely used in the control of interconnected power systems, distribution networks, traffic systems, computer and communication networks, civil structure systems, and aerospace systems [7], [8], [15], [16]. Considerable research effort was made for decentralized control for large scale systems, [17] studied the stability of decentralized control of interconnected systems using adaptive control scheme. Stability analysis of networked systems was studied by [18] using observer based decentralized control scheme. Reference [16] discussed optimal control design for decentralized large scale systems using modified LQR control. Optimal stabilization for decentralized linear systems using output feedback control strategy was studied by [13]. Stability of decentralized singularly perturbed systems was discussed by [19] and a robust controller was designed using a unified approach. An output feedback control scheme for discrete time decentralized singularly perturbed systems was developed by [20] using an iteration method.

The investigated model in this research of a large scale stochastic decentralized interconnected system was a reduced order quasi-steady state model of the original system using singular perturbation techniques. The minimization of conditions and constraints reaches a solution which applies Lyapunov equations coupled with constraints equations to optimize the performance index of the reduced-order model.

\section{PROBLEM STATEMENT}

Consider the linear time-invariant decentralized singularlyperturbed model in (1) representing the system shown in Fig. 1. 


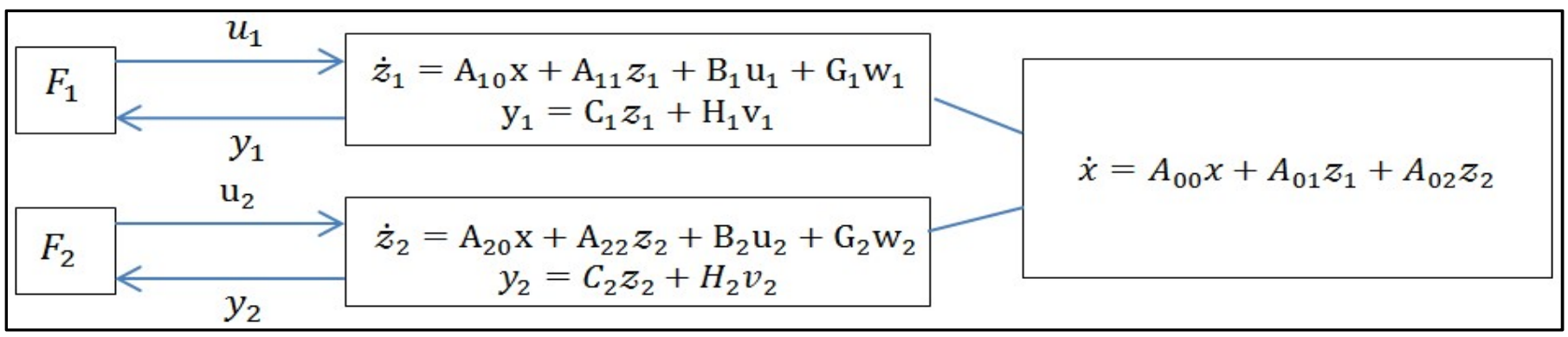

Fig.1. Decentralized system setup with two subsystems

$$
\begin{aligned}
\dot{x} & =A_{00} x+A_{01} z_{1}+A_{02} z_{2} \\
\varepsilon \dot{z}_{i} & =A_{i 0} x+A_{i i} z_{i}+B_{i} u_{i}+G_{i} w_{i} \\
y_{i} & =C_{i} z_{i}+H_{i} v_{i}, \quad i=1,2
\end{aligned}
$$

where $x \in \mathcal{R}^{n}$ and $z_{i} \in \mathcal{R}^{m_{i}}$ are the state variables of the slow main system and fast subsystem $z_{i}$. The input $u_{i} \in$ $\mathcal{R}^{r_{i}}$ is the control input vector, and the output $y_{i} \in \mathcal{R}^{p_{i}}$ is the control output vector of subsystem $i$. $w_{i}$ and $v_{i}$ are the process and measurement noises respectively, $G_{i}$ and $H_{i}$ are constant scaling matrices corresponds to noise for subsystem i. $A_{00}, A_{0 i}, A_{i 0}, A_{i i}, B_{i}$, and $C_{i}$ are constant matrices with appropriate matching dimensions(for $i=1,2$ ). It is assumed that the singular perturbation parameter $0<\varepsilon \ll 1$ is a small positive number.

The objective of this research is to develop a stabilizing, robust to noise and optimal control of reduced-order output feedback controller. This controller should be able to stabilize a linear time-invariant stochastic singularlyperturbed system via decentralized control. Besides states unavailability, the lack of direct control to the main system is a major issue in the problem formulated in (1). The criterion to be analyzed is a standard LQR performance index. The proposed technique will use singular perturbation approach to reduce the order of the original model to be applicable to large-scale systems.

\section{MAIN RESULTS}

In singular perturbation, when $\varepsilon$ approaches zero, we assume that the fast subsystems state variables, $z_{1}$ and $z_{2}$ have reached quasi-steady state. Hence, the system order is reduced to the order of the main system, which is equal to the dimension of the slow state variable $x$. In Other words, when $\varepsilon \rightarrow 0$, then $\varepsilon \dot{z}=0$, which means that only the slow part dynamics affect the system. Now, let $\overline{\mathrm{x}}, \bar{z}$ and $\overline{\mathrm{u}}$ represent the quasi-steady state variables of the system. The slow variables of the system can be set as: $x^{s}=\bar{x}, u^{s}=$ $\bar{u}$ and $y^{s}=\bar{y}$. Then (1)reduces to

$$
\begin{aligned}
\dot{x}^{S} & =A_{00} x^{S}+\sum_{i} A_{0 i} \bar{z}_{i} \\
0 & =A_{i 0} x^{S}+A_{i i} \bar{z}_{i}+B_{i} u_{i}^{S}+G_{i} w_{i} ; i=1,2 \\
y_{i}^{S} & =C_{i} \bar{z}_{i}+H_{i} v_{i}
\end{aligned}
$$

The second algebraic equation of system (2) can be inverted for $\bar{z}_{\mathrm{i}}$, resulting in:

$$
\bar{z}_{i}=-A_{i i}^{-1}\left(A_{i 0} x^{s}+B_{i} u_{i}^{s}+G_{i} w_{i}\right) ; \quad i=1,2
$$

where $A_{i i}$ are non-singular matrices. Theninserting $\bar{z}_{i}$ from (3) into the dynamic part and third equation of (2)yielding the reduced order system

$$
\begin{aligned}
& \dot{x}^{s}=\tilde{A} x^{s}+\sum_{1}^{2} \tilde{B}_{i} u_{i}^{s}+\tilde{G}_{i} w_{i} \\
& y_{i}^{s}=\tilde{C}_{i} x^{s}+\widetilde{D}_{i} u_{i}^{s}+\hat{G}_{i} w_{i}+H_{i} v_{i} ; i=1,2
\end{aligned}
$$

where,

$$
\begin{gathered}
\tilde{A}=A_{00}-A_{01} A_{11}^{-1} A_{10}-A_{02} A_{22}^{-1} A_{20} \\
\tilde{B}_{i}=-A_{0 i} A_{i i}^{-1} B_{i} \\
\tilde{C}_{i}=-C_{i} A_{i i}^{-1} A_{i 0} \quad \widetilde{D}_{i}=-C_{i} A_{i i}^{-1} B_{i} \\
\tilde{G}_{i}=-A_{0 i} A_{i i}^{-1} G_{i} \text { and } \widehat{G}_{i}=-C_{i} A_{i i}^{-1} G_{i}
\end{gathered}
$$

For subsystem $i$, the performance $\operatorname{index} J_{i}$ is given by

$$
\begin{array}{r}
J_{i}=\frac{1}{2} E\left\{\int _ { 0 } ^ { \infty } \left(\left[x^{S}\right]^{T} Q_{x} x^{S}+\bar{z}_{i}^{T} Q_{z i} \bar{z}_{i}\right.\right. \\
\left.\left.+\left[u_{i}^{S}\right]^{T} R_{i} u_{i}^{S}\right) d t\right\}
\end{array}
$$

where: $Q_{x}, Q_{z_{i}}$ are positive semi definite symmetric matrices and $R_{i}$ is a symmetric positive definite matrix. By substituting the values of $\bar{z}_{\mathrm{i}}$ in $\quad$ (3) we get the reduced performance index as

$$
\begin{aligned}
J_{i}=\frac{1}{2} E\left\{\int _ { 0 } ^ { \infty } \left(\left[x^{S}\right]^{T} \hat{Q} x^{s}\right.\right. \\
+\sum_{i=1}^{2}\left\{\left[u_{i}^{S}\right]^{T} R_{c i} u_{i}^{S}\right. \\
\left.\left.\left.+2\left[x^{S}\right]^{T} N_{i} u_{i}^{s}\right\}\right) d t\right\}+J_{w}
\end{aligned}
$$

where $J_{w}$, is a term contains the integral of the variance of the noise which is not affected by control [21], and

$$
\begin{gathered}
\hat{Q}=Q_{x}+\sum_{1}^{2}\left[A_{i 0}^{T} A_{i i}^{-T} Q_{z i} A_{i i}^{-1} A_{i 0}\right] \\
N_{i}=B_{i}^{T} A_{i i}^{-T} Q_{z i} A_{i i}^{-1} A_{i 0}
\end{gathered}
$$




$$
R_{c i}=R_{i}+B_{i}^{T} A_{i i}^{-T} Q_{z i} A_{i i}^{-1} B_{i}, \quad i=1,2
$$

The static output feedback input $u_{i}^{s}$ for each subsystem $i$ can be written as

$$
u_{i}^{s}=F_{i} y^{s}=\Omega_{i} u_{0 i}^{s}+\Omega_{i} F_{0 i} \tilde{C}_{i} x^{s}+\widetilde{w}_{i}
$$

where,

$$
\begin{gathered}
\widetilde{w}_{i}=\Omega_{i} F_{0 i} \widehat{G}_{i} w_{i}+\Omega_{i} F_{0 i} H_{i} v_{i} \\
\Omega_{i}=\left(I-F_{0 i} \widetilde{D}_{i}\right)^{-1}
\end{gathered}
$$

The system and performance index of the closed loop system are shown in (11) and (12) respectively

$$
\begin{gathered}
\dot{x}^{s}=A_{c} x^{s}+\widehat{w} \\
J_{i}=\frac{1}{2} E\left\{\int_{0}^{\infty}\left(\left[x^{s}\right]^{T} Q_{c} x^{s}\right) d t\right\}+J_{\widehat{w}}
\end{gathered}
$$

where $J_{\widehat{w}}$, is a term not affected by control and,

$$
\begin{gathered}
A_{c}=A_{r}-\widetilde{B}_{1} F_{01} \tilde{C}_{1}-\widetilde{B}_{2} F_{02} \tilde{C}_{2} \\
A_{r}=\tilde{A}-\widetilde{B}_{1} R_{c 1}^{-1} N_{1}^{T}-\widetilde{B}_{2} R_{c 2}^{-1} N_{2}^{T} \\
\widehat{W}=\widetilde{B}_{1} \widetilde{w}_{1}+\widetilde{B}_{2} \widetilde{w}_{2} \\
Q_{c}=Q_{r}+\tilde{C}_{1}^{T} F_{01}^{T} R_{c 1} F_{01} \tilde{C}_{1}+\tilde{C}_{2}^{T} F_{02}^{T} R_{c 2} F_{02} \tilde{C}_{2} \\
Q_{r}=\hat{Q}+N_{1} R_{c 1}^{-1} N_{1}^{T}+N_{2} R_{c 2}^{-1} N_{2}^{T} \\
\hat{R}_{c i}=\Omega_{i}^{T} R_{c i} \Omega_{i}
\end{gathered}
$$

The solution of the performance index for this system found to be

$$
J_{i}=\frac{1}{2} \operatorname{tr}\left(P_{i} \Sigma\right)
$$

where $\Sigma=E\left\{x(0) x^{T}(0)\right\}$ and the symmetric $P_{i} \geq 0$ is the solution of

$$
0=A_{c}^{T} P_{i}+P_{i} A_{c}+Q_{c} \equiv K_{i}
$$

Because of the decentralized setup of the system, the two subsystems dynamics are uncoupled. The objective is to findan optimal control $u_{i}$ for each subsystem $i$ that is able to minimize the common performance index. To minimize (14) satisfying (15) we introduced the Hamiltonian function (16) and a symmetric positive definite slack variable $Z_{i}$

$$
H_{i}=\operatorname{tr}\left(P_{i} \Sigma\right)+\operatorname{tr}\left(K_{i} Z_{i}\right)
$$

Minimization by setting the partial derivatives of (16) with respect to $Z_{i}, P_{i}$, and $F_{0 i}$ to zero, we get

$$
\begin{gathered}
0=\Sigma+A_{c} Z_{i}+Z_{i} A_{c}^{T} \\
0=A_{c}^{T} P_{i}+P_{i} A_{c}+Q_{c}
\end{gathered}
$$

$$
0=R_{c i i} F_{0 i} \tilde{C}_{i} Z_{i} \tilde{C}_{i}^{T}-\widetilde{B}_{i}^{T} P_{i} Z_{i} \tilde{C}_{i}^{T}
$$

Feedback gain can be calculated using equation (19) in the form

$$
F_{0 i}=R_{c i i}^{-1} \tilde{B}_{i}^{T} P_{i} Z_{i} \tilde{C}_{i}^{T}\left(\tilde{C}_{i} Z_{i} \tilde{C}_{i}^{T}\right)^{-1}
$$

Numerical iterative solution of equations (17), (18) and (20) in the same order, converges to a solution for theoptimal feedback gain $F_{0 i}$. The initial value of $F_{0 i}$ can be selected randomly making sure it maintain the stability of the close loop system.

\section{NUMERICAL EXAMPLE}

Consider the following decentralized singularly perturbed system:

$$
\begin{gathered}
\dot{x}=\left[\begin{array}{cc}
-2 & 0 \\
0 & -3
\end{array}\right] x+\left[\begin{array}{cc}
1 & -1 \\
-1 & -2
\end{array}\right] z_{1}+\left[\begin{array}{cc}
-3 & 2 \\
-4 & -1
\end{array}\right] z_{2} \\
\varepsilon \dot{z}_{1}=\left[\begin{array}{ll}
-1 & 2 \\
-4 & 3
\end{array}\right] x+\left[\begin{array}{cc}
-5 & 4 \\
-3 & -1
\end{array}\right] z_{1}+\left[\begin{array}{l}
1 \\
2
\end{array}\right] u_{1}+\left[\begin{array}{l}
0.1 \\
0.1
\end{array}\right] w_{1} \\
\varepsilon \dot{z}_{2}=\left[\begin{array}{ll}
-1 & 5 \\
-3 & 2
\end{array}\right] x+\left[\begin{array}{cc}
-2 & -1 \\
-2 & -3
\end{array}\right] z_{2}+\left[\begin{array}{l}
1 \\
2
\end{array}\right] u_{2}+\left[\begin{array}{l}
0.1 \\
0.1
\end{array}\right] w_{2} \\
y_{2}=\left[\begin{array}{ll}
1 & 0
\end{array}\right] z_{2}+v_{2} \\
y_{1}=\left[\begin{array}{ll}
0 & 1
\end{array}\right] z_{1}+v_{1}
\end{gathered}
$$

with ones as the expected value of the initial condition of the states, $\varepsilon=0.1$ and only $z_{1}$ the optimal full order model feedback gain found to be:

$F^{*}=\left[\begin{array}{llllll}-0.16 & -0.27 & 0.04 & 0.46 & -0.04 & 0.05\end{array}\right]$,

with

$$
K(0)=\left[\begin{array}{rccccc}
0.41 & -0.12 & 0.02 & -0.02 & -0.05 & 0.02 \\
-0.12 & 0.46 & 0 & -0.01 & 0.05 & -0.05 \\
0.02 & 0 & 0.01 & 0 & 0 & 0 \\
-0.02 & -0.01 & 0 & 0.03 & 0 & 0 \\
-0.05 & 0.05 & 0 & 0 & 0.04 & -0.02 \\
0.02 & -0.05 & 0 & 0 & 0.02 & 0.03
\end{array}\right]
$$

and the close loop poles $(p)$ are

$$
p=\left[\begin{array}{l}
-33.68+j 29.19 \\
-33.68-j 29.19 \\
-34.99 \\
-11.08 \\
-5.59+j 9.00 \\
-5.59-j 9.00
\end{array}\right]
$$

with $W_{1}=W_{2}=0.1$, Full order system optimal cost is

$$
J_{1}^{*}=0.3150
$$

The reduced order model

$$
\dot{x}^{s}=\left[\begin{array}{cc}
-4 & -12.46 \\
4 & -16.38
\end{array}\right] x^{s}+\left[\begin{array}{c}
0.12 \\
-1.35
\end{array}\right] u_{1}^{s}+\left[\begin{array}{c}
0.02 \\
-0.05
\end{array}\right] w_{1}
$$




$$
x^{s}=\left[\begin{array}{ll}
-1 & 0.53
\end{array}\right] x^{s}+0.41 u_{1}^{s}+0.01 w_{1}+v_{1}
$$

The optimal feedback gain for this reduced order model was

$$
F_{1}^{*}=0.0316
$$

By applying this optimal feedback gain to the full order model, the closed loop system optimal cost is

$$
J_{1}^{*}=0.3321
$$

and the final P calculated for the closed loop system

$$
\begin{aligned}
& P(0) \\
& =\left[\begin{array}{rrrrrr}
0.47 & -0.14 & 0.03 & -0.03 & -0.05 & 0.03 \\
-0.14 & 0.49 & 0 & -0.01 & 0.05 & -0.05 \\
0.03 & 0 & 0.01 & -0.01 & 0 & 0 \\
-0.03 & -0.01 & -0.01 & 0.03 & 0 & 0 \\
-0.05 & 0.05 & 0 & 0 & 0.04 & -0.02 \\
0.03 & -0.05 & 0 & 0 & -0.02 & 0.03
\end{array}\right]
\end{aligned}
$$

The closed loop system poles $(p)$ are

$$
p=\left[\begin{array}{l}
-28.74+j 28.33 \\
-28.74-j 28.33 \\
-34.95 \\
-4.86+j 9.18 \\
-4.86-j 9.18 \\
-12.85
\end{array}\right]
$$

The response of the output and states of the system are shown in Figs. 2 and 3 respectively. The optimal cost of the reduced order model is very close to that of the full order system.

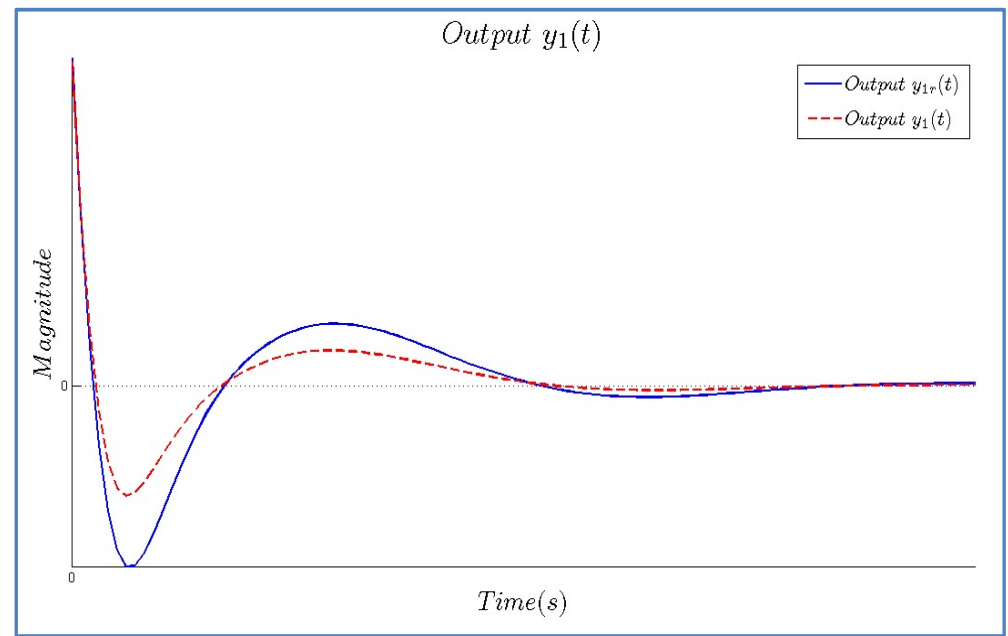

Fig.2. Output response for full and reduced order Models

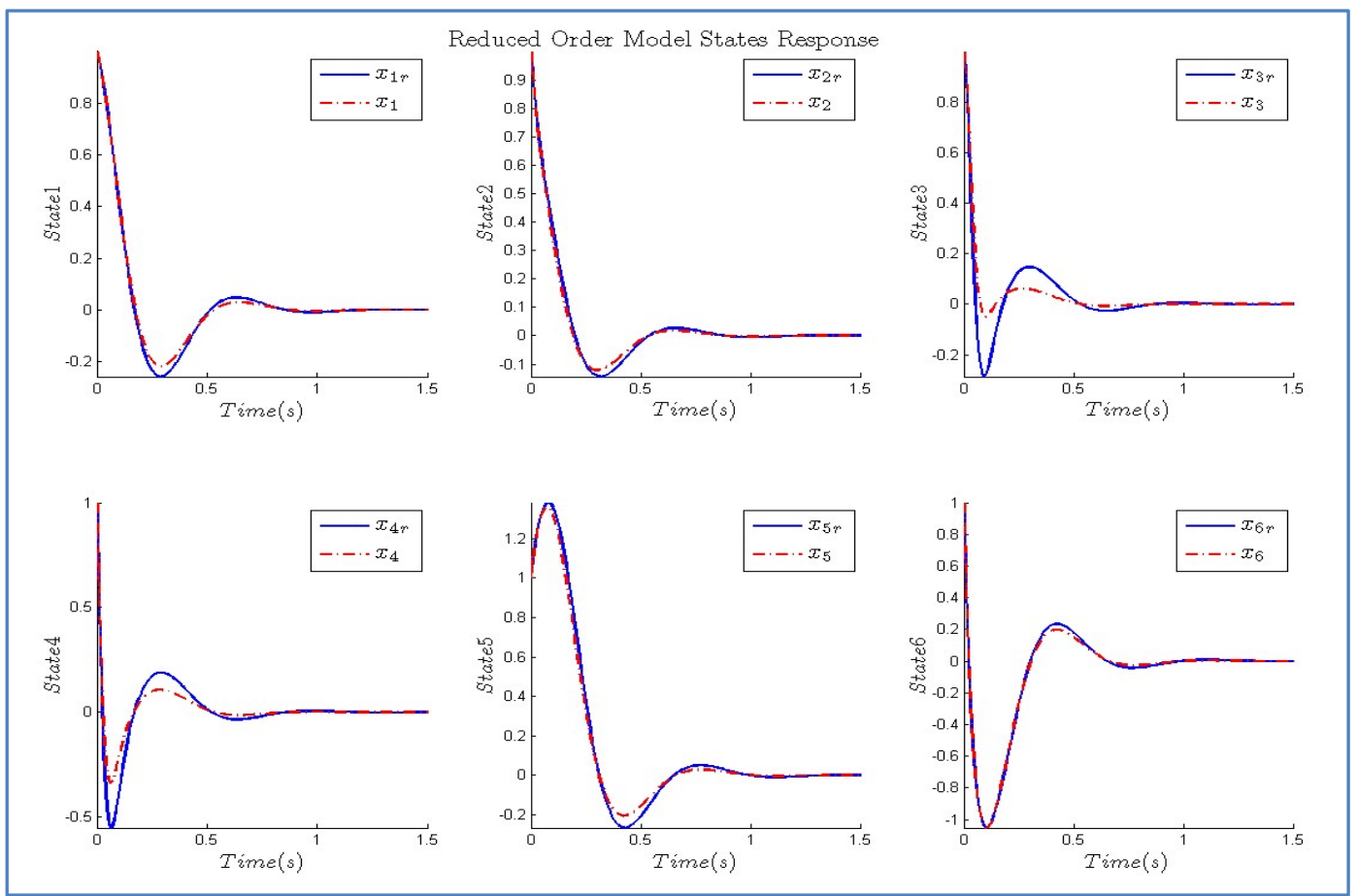

Fig.3. State response for full and reduced order Models 


\section{CONCLUSIONS}

In this paper, the singularly perturbed decentralized system was found to have an optimal control with optimum feedback gain satisfying the constrained Lyapunov equations in the quasi-steady state model. When the system is exposed to either a measurement noise, input disturbance or both, a solution exists using numerical iterative technique with certain assumptions in addition to basic knowledge of the white noise distribution. The numerical example has shown excellent results that the proposed method has succeeded in decreasing the computational workspace and the quadratic convergence has been attained. The new technique in this research will help to simplify system analysis and controller design without the need of states availability or observer design. The algorithm used for two subsystems, but it can be expanded for multi-subsystems.

\section{REFERENCES}

[1]. P. Kokotovic, H. Khalil and J. O'Reilly, Singular Perturbation Methods in Control: Analysis and Design, London: Academic Press, 1986.

[2]. J. Leigh, Control Theory, London: The Institution of Engineering and Technology, 2004.

[3]. N. Jagan, Control Systems, Hyderabad, India: BS Publications, 2007.

[4]. S. Engelberg, A Mathematical Introduction to Control Theory, London: Imperial College Press, 2005.

[5]. J. Speyer and D. Jacobson, Primer on Optimal Control Theory, Philadelphia: Society for Industrial and Applied Mathematics, 2010.

[6]. M. Golnaraghi, B. C. and M. Golnaraghi, Automatic Control Systems, Hoboken, NJ: John Wiley \& Sons. Inc., 2010.

[7]. N. Sandell, P. Varaiya, M. Athans and M. Safonov, "Survey of decentralized control methods for large scale systems," IEEE Transactions on Automatic Control, vol. 23, no. 2, pp. 108-128, 1978.

[8]. D. Zhang, X. Wang, and L. Meng, "Consensus problems for high-order LTI systems: a decentralized static output feedback method." International Journal of Innovative Computing, Information and Control, pp. 2143-2154, 2013.

[9]. V. Seksena, J. O'Reilly and P. Kokotovic, "Singular perturbations and time-scale methods in control theory: Survey 1976-1983," Automatica, vol. 20, no. 3, pp. 273-293, 1984.

[10].Y. Zhang, D. Naidu, C. Cai and Y. Zou, "Singular perturbations and time scales in control theories and applications: an overview 202-2012," Internation Journal of Information and Systems Sciences, vol. 9, no. 1, pp. 1-36, 2014.

[11].D. Naidu, "Singular perturbations and time scales in control theory and applications: an overview," Dynamics of Continuous Discrete and Impulsive Systems Series B, vol. 9, no. 2, pp. 233-278, 2002.

[12].K. Kalsi, Decentralized Observer-Based Control of Uncertain Dynamic Systems, West Lafayette, IN: Diss. Purdue University, 2010.
[13]. Savkin and I. Petersen, "Optimal Stabilization of Linear Systems via Decentralized Output Feedback," IEEE Transactions on Automatic Control, vol. 43, no. 2, pp. 292 - 294, 1998.

[14].Anderson and J. Moore, "Time-varying feedback laws for decentralized control," IEEE Transactions on Automatic Control, vol. 26, no. 5, pp. 1133-1139, 1981.

[15].J. Marden and J. Shamma, "Game theory and distributed control" In: P. Young, S. Zamir (editors), Handbook of game theory, Vol.4, Elsevier Science, 2012.

[16].J. Lynch and K. Law, "Decentralized control techniques for large-scale civil structural systems" Proc. of the 20th Int. Modal Analysis Conference (IMAC XX), Los Angeles, CA, USA, February 2002.

[17].P. Ioannou, "Decentralized adaptive control of interconnected systems." IEEE Transactions on Automatic Control, vol. 31, no. 4, pp. 291-298, 1986.

[18].Elmahdi, A. Taha, D. Sun and J. Panchal, "An Optimal General Purpose Scheduler for Networked Control Systems." IEEE International Conference on Systems, Man and Cybernetics (SMC), pp. 234-239, 2014.

[19].Hyun, M.E. Sawan, D.G. Lee, and D. Kim "Robust stability for decentralized singularly perturbed unified system." Proceedings of American Control Conference. 2006.

[20].Yao, "An iteration method to sub-optimal output feedback computer control of decentralized singularlyperturbed systems." Innovative Computing, Information and Control, 2007. ICICIC'07. Second International Conference on. IEEE, 2007.

[21].Haddad and P. Kokotovic, "Stochastic control of linear sigularly perturbed systems," IEEE Transactions on Automatic Control, vol. 22, no. 5, pp. 815-821, 1977. 